\title{
MIELINÓLISE PONTINA E EXTRA-PONTINA ASSOCIADA A SHOSHIN BERIBÉRI EM PACIENTE ETILISTA
}

\author{
Aluska C. Aguiar1, Vanessa M. Costa², Paulo C. Ragazzo³, Roberto R. Caiado4, \\ Carlos A. Gusmão ${ }^{5}$, Sebastião Eurico de Melo-Souza ${ }^{6}$
}

\begin{abstract}
RESUMO - A mielinólise pontina está classicamente associada à rápida correção de hiponatremia crônica. Recentemente, fatores importantes adicionais tem sido descritos na patogênese dessa condição. Relatamos o caso de um paciente de 43 anos, etilista, desnutrido, que apresentou quadro agudo de insuficiência cardíaca por "Shoshin beribéri", insuficiência renal com tratamento por diálise. Evoluiu com tetraparesia e coma. Apresentou mielinólise pontina central e extra-pontina à ressonância magnética de crânio e anormalidades no potencial evocado auditivo.
\end{abstract}

PALAVRAS-CHAVE: mielinólise pontina e extra-pontina, alcoolismo, Shoshin beribéri.

\begin{abstract}
Central pontine and extra-pontine myelinosis in alcoholic patient with Shoshin beriberi
ABSTRACT - The central pontine myelinosis is classically related with rapid correction of chronic hyponatremia. Recently, important aditional factors have been described in the pathogenesis of this condition. We report the case of a 43-year-old alcoholic malnourished man, with acute renal failure with dialytic treatment, and output failure Shoshin beriberi. He had tetraplegy and coma. Confirmation of pontine and extrapontine myelinosis by magnetic resonance imaging, and abnormalities on auditive evoked potentials are described.

KEY WORDS: central pontine and extra-pontine myelinosis, alcoholism, Shoshin beriberi.
\end{abstract}

Mielinólise pontina (MP) é enfermidade desmielinizante do encéfalo que acomete principalmente a região da ponte, mas que pode atingir também regiões extra-pontinas (MEP), Está classicamente associada à rápida correção de hiponatremia crônica. Recentemente, evidências de fatores adicionais significativos para a patogênese da doença têm sido relacionados. Um subgrupo particularmente predisponente para desenvolver MP é o de etilistas crônicos.

Relatamos um caso de MP em MEP.

\section{CASO}

Homem de 43 anos, com história de etilismo há 10 anos, tabagismo e hiporexia há 1 ano. Há alguns meses apresentou alteração de comportamento e declínio cognitivo. Uma semana antes da internação começou a apresentar tosse, dispnéia e edema de membros inferiores. Foi admitido neste Serviço em choque cardiogênico, anúria por insuficiência renal aguda (IRA) e edema agudo de pulmão. Evoluiu com choque refratário ao uso de dopamina e noradrenalina. Fez eco-Doppler que demonstrou funções sistólicas e diastólicas normais, com FE: $60 \%$. Foi feita a hipótese diagnóstica de "Shoshin" beribéri. Fez teste terapêutico com vitamina B1 endovenosa, recuperando-se do quadro de choque cardiogênico rapidamente. Para IRA, foi necessária diálise tendo recuperado completamente a função renal ao final do tratamento. Evoluiu com coagulação intravascular disseminada, com plaquetopenia de consumo e elevação de d-dímeros. Teve parada cardio-respiratória com imediata reanimação, mas permaneceu em estado vegetativo persistente, com ciclo sono-vigília. Está atualmente em programa de reabilitação motora.

O estudo do líquor não mostrou alterações infecciosas, ou inflamatórias. Foi admitido com sódio sérico de $136 \mathrm{meq} / \mathrm{l}$, sofrendo oscilação até $160 \mathrm{meq} / \mathrm{l}$, durante a diálise, sem correção eletrolítica. Sódio permaneceu após esse episódio em torno de $140 \mathrm{meq} / \mathrm{l}$.

Eletrencefalograma- Disfunção córtico-subcortical contínua severa, difusa, não específica e depressão da atividade elétrica cerebral contínua e difusa.

Ressonância magnética do crânio - lesão arredondada com hipersinal em T2 e Flair na porção central da ponte e adjacente ao IV ventrículo. Lesões arredondadas com as mesmas características de sinal foram identificadas na face mediana dos pedúnculos cerebelares médios de ambos os lados, adjacente ao IV ventrículo. Essas alterações foram sugestivas de mielinólise pontina/extrapon-

Instituto de Neurologia de Goiânia, Goiânia GO, Brasil (ING): ${ }^{1}$ Neurologista; ${ }^{2}$ Neurologista; ${ }^{3}$ Neurofisiologista; ${ }^{4}$ Cirurgião torácico; ${ }^{5}$ Cardiologista; ${ }^{6}$ Neurologista, Coordenador de Ensino e Pesquisa.

Recebido 9 Junho 2003, recebido na forma final 28 Janeiro 2004. Aceito 9 Março 2004.

Dr. Aluska C. Aguiar INGO - Praça Gilson Alves de Souza 140 / Setor Bueno - 74210-250 Goiânia GO - Brasil. 


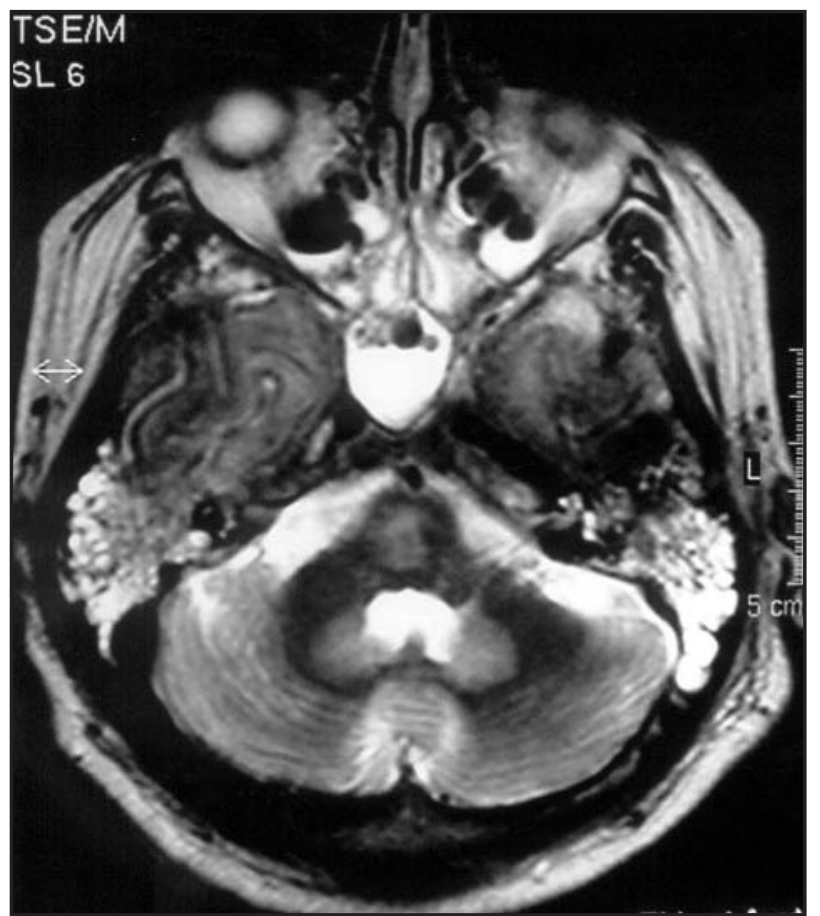

Fig 1. Mielinólise Pontina e Extra-Pontina associada a Shoshin Beribéri.

tina. Hematoma subagudo lobar frontal esquerdo. Tênue hipossinal linear na superfície cortical de ambos os hemisférios cerebrais, podendo representar necrose laminar(Figs 1-3).

Potencial evocado auditivo do tronco cerebral (PEATC). Foram registrados as ondas I-IV e V bilateralmente, com latências absolutas e intervalos interpicos I-V dentro dos limites da normalidade. Nota-se ausência da onda III bilateralmente, o que pode representar artefatos, ou efeitos de lesão pontina parcial bilateral. O exame de PEATC mostra sinais de disfunção das vias auditivas centrais ao nível pontino no tronco cerebral, bilateralmente .

SPECT - Evidencia-se área de ausência de metabolismo em todo sistema nervoso central, persistindo apenas discreta perfusão na fossa posterior.

\section{DISCUSSÃO}

Mielinólise pontina foi descrita por Victor, Adams e Mancall, em 19491. Um dos principais estímulos para a ocorrência de mielinólise pontina é a rápida correção de sódio em pacientes hiponatrêmicos. Mas foi também relacionada à terapia com diuréticos ${ }^{2}$, transplante renal ${ }^{3}$, consumo alcoólico excessivo e após transplante hepático,em indivíduos desnutridos com hipotermia, diabetes mellitus descontrolado, câncer e doença hematológica maligna ${ }^{4}$. A correção rápida do sódio sérico para níveis superiores a $130 \mathrm{mEq} / \mathrm{l}$ dentro das primeiras 48 horas de terapia, ou sua elevação acima de $12 \mathrm{mEq} / \mathrm{l}$ nas primeiras 24 horas, relacionase com lesões cerebrais desmielinizantes. Uma

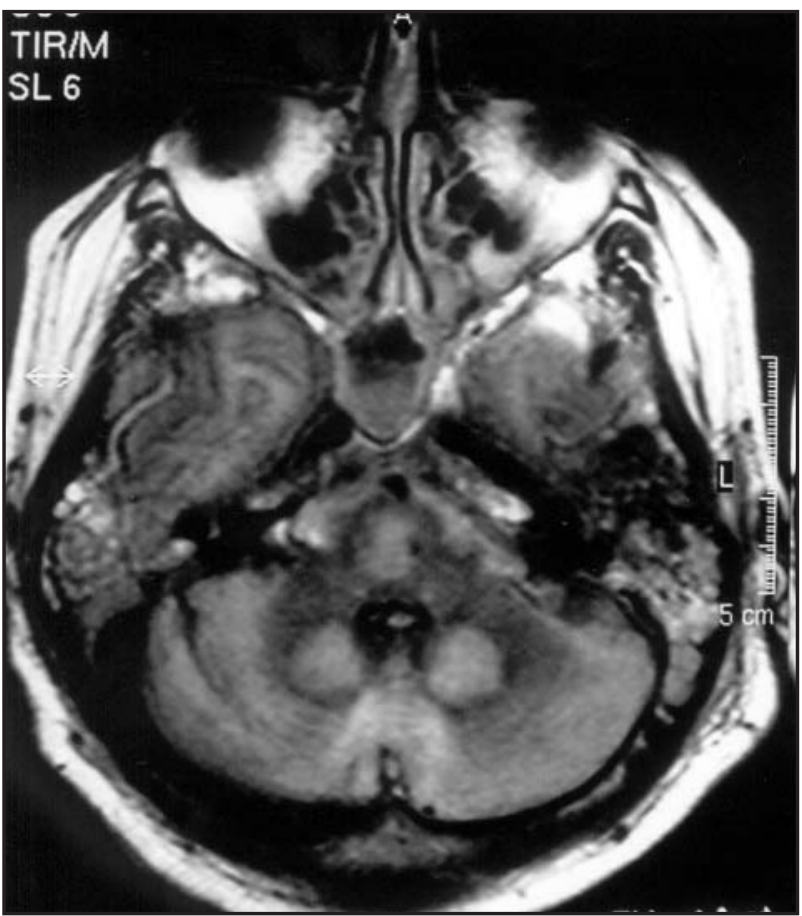

Fig 2. Mielinólise Pontina e Extra-Pontina associada a Shoshin Beribéri.

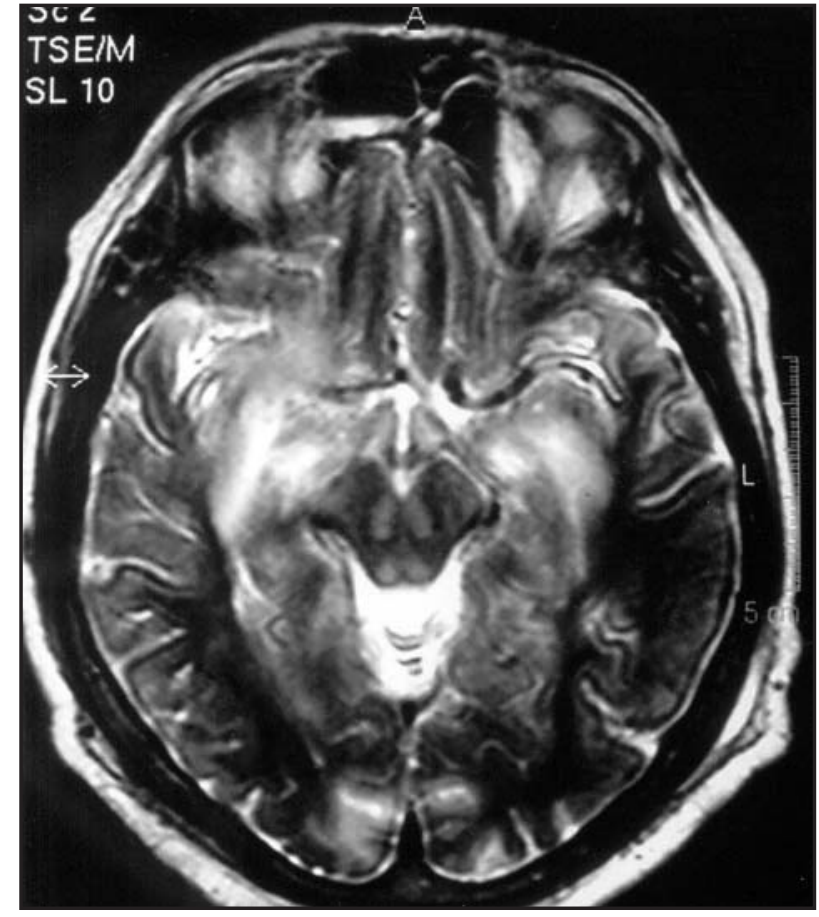

Fig 3. Mielinólise Pontina e Extra-Pontina associada a Shoshin Beribéri.

abordagem adequada sugerida é repor o déficit de sódio sérico de 0,5 a $1 \mathrm{mEq} / \mathrm{l} / \mathrm{h}$ até a redução dos sintomas e não exceder $130 \mathrm{mEq} / \mathrm{l}$ de sódio sérico nas primeiras 48 horas $^{5}$. 
A razão de se evitar rápida correção de sódio seria pela hipótese osmótica, implicando em redução da habilidade das células em condições hipoosmóticas em responder a rápidos aumentos de sódio extracelular, levando a desidratação celular e eventual morte da célula. Condições como alcoolismo crônico e desnutrição são fortes indicadores independentes da ocorrência da doença. É inclusive descrita a ocorrência da doença com reposição lenta do sódio em paciente etilista, descartando a exigência da reposição rápida de sódio para MP6 . Pacientes etilistas e com falência hepática têm suporte de glicose reduzido, sobrevivendo em condições metabólicas precárias, o que é agravado na ausência de tiamina, que diminui o aporte de glicose cerebral. Qualquer estresse metabólico extra proporciona risco adicional para a glia desses pacientes ${ }^{6}$.

O quadro clínico da MP inclui: mutismo, tremor, incontinência, convulsões, anormalidades pupilares e oculomotoras, síndrome "locked-in", anormalidades de reflexos córtico-espinhais e fadiga 6 . Pacientes desenvolvem paralisia pseudobulbar tardia (2 a 6 dias), tetraparesia espástica, torpor e coma. Nos casos em que a MP/MEP resulta da correção rápida da hiponatremia, a doença apresenta caráter bifásico. Inicialmente prevalecem os sintomas primários da encefalopatia causada pela hiponatremia em si. Na chamada segunda fase, há estabelecimento da síndrome neurológica descrita acima, 2 a 3 dias após a correção do sódio.

A hipótese diagnóstica de MP pode ser confirmada pelos exames de neuroimagem. A tomografia computadorizada mostra áreas de hipodensidade na ponte, ou ainda extra-pontinas, em geral comprometendo a base da ponte. E a ressonância magnética de crânio mostra sinal hipointenso em T1 e hiperintenso em T2. Anormalidades em potenciais evocados visuais foram descritos ${ }^{9}$. As respostas evocadas auditivas do tronco cerebral podem mostrar latências III-IV e I-V prolongadas, consistentes com lesões pontinas. O eletrencefalograma pode mostrar lentificação e baixa voltagem. Os níveis de proteína mielínica básica no líquor podem estar aumentados.

As preparações histológicas mostram um padrão típico, que consiste em uma lesão triangular invertida. Embora denominada mielinólise, algum grau de lesão axonal pode ser identificado. Esta lesão apresenta uma área central e outra periférica, ou marginal. Neurônios e axônios estão mais preser- vados na periferia da lesão. O envolvimento extrapontino é visto de $10-50 \%$ dos casos, com palidez difusa da susbstância branca cerebelar, susbstância cinzenta periaquedutal e substância negra. Pode haver também lesão do tálamo, gânglios da base e córtex cerebral ${ }^{4}$.

Com relação à prevenção da doença, pacientes de alto risco devem ser identificados quanto à sua alta ingesta de álcool e deficiência nutricional. Deve-se evitar estresse metabólico, com reposição de vitaminas como tiamina à admissão, e se necessária a correção do sódio, fazê-la muito lentamente. Não há tratamento específico para doença já instalada.

"Shoshin" beribéri é caracterizada como forma fulminante de insuficiência cardíaca devida a deficiência de tiamina com circulação hiperdinâmica e falência cardíaca de alto débito do tipo agudo ${ }^{10}$. "Shoshin" tem uma apresentação diferente da forma clássica da falência cardíaca por beribéri. Caracteriza-se por extremidades frias, pressão sanguínea baixa, acidose metabólica importante ${ }^{11}$ e falência biventricular, podendo os pacientes cursarem com oliguria e disfunção tubular renal. A ecocardiografia pode ser normal apesar da hipocinesia e dilatação ventricular esquerda poder ser notada. Não há sinais específicos no eletrocardiograma nos pacientes acometidos. O diagnóstico é suspeitado em pacientes com alcoolismo crônico que desenvolvem falência cardíaca global aguda ${ }^{13}$. No tipo ocidental de beribéri, que é encontrado quase exclusivamente em alcóolatras, a síndrome pode não apresentar débito cardíaco alto. Essas diferenças podem ser atribuídas a cardiomiopatia alcoólica ${ }^{14}$. A melhora clínica é dramática após a infusão de vitamina B1 intravenosa. O estado hiperdinâmico resolve-se em horas e o aumento pressórico é imediato ${ }^{10}$. O nosso caso confirma a efetividade da infusão venosa da tiamina para "Shoshin" e a ineficácia das drogas vasoconstrictoras, conforme relatado por Delorme e col. em $1986^{16}$.

$O$ relato deste caso mostra o achado de MP e MEP, em etilista com história de desnutrição, insuficiência cardíaca descompensada por "Shoshin" beribéri e insuficiência renal aguda, tendo sido necessários diálise e altas doses de diuréticos. Esses achados reforçam a natureza multifatorial da doença. A mielinólise foi confirmada pela ressonância magnética de crânio, e o acometimento pontino foi identificado ainda pelo potencial evocado auditivo. $O$ estado vegetativo do paciente pode ter sido agravado por encefalopatia pós-anóxia. 


\section{REFERÊNCIAS}

1. Victor M, Adams RD, Mancall EL. Central pontine myelinosis: a hitherto undescribed disease occurring in alcoholic and malnourished patients. Arch Neurol Psychiatry 1959;81:154-172.

2. Cambier J, Masson M, Dairou R, Gray F, Henin D, Laurent D. Centropontine myelinosis associated with diffuse lesions of the white matter: water-electrolyte disturbances associated with diuretic therapy. J Neurol 1977;133 89-99.

3. Mahoney CA, Arieff A. Uremic encephalopathies: clinical, biochemical, and experimental features. Am J Kidney Dis 1982;2:324-336.

4. Raffin CN. Mielinólise central pontina. In Melo-Souza SE (ed). Tratamento das doenças neurológicas. Rio de Janeiro: Guanabara Koogan, 2000:570-571.

5. Santos BFC, Andrei AM, Rodrigues MJ. Distúrbios na concentração plasmática de sódio In Knobel E (ed). Condutas no paciente grave. São Paulo: Atheneu. 1999:447-452.

6. Ashrafian H, Davey P. A review of the causes of central pontine myelinosis: yet another apoptotic illness? Eur J Neurol 2001;8:103-109.

7. Germiani FMB, Roriz M, Nabhan SK, Teive HAG, Werneck LC. Mielinólise pontina central e extra-pontina em paciente alcoolista sem distúrbios hidro-eletrolíticos. Arq Neuropsiquiatr 2002;60:1030-1033.
8. Bernardini GL, Mancal EL. Mielinólise central pontina In Merrit HN. Tratado de neurologia. Rio de Janeiro: Guanabara Koogan, 2002:689-691.

9. Dickoff DJ, Raps M, Yahr MD. Sriatal syndrome following hyponatremia and its rapid correction, a manifestation of extrapontine myelinosis confirmed by magnetic resonance imaging. J Neurol 2003;250:103-105.

10. Naidoo DP, Rawat R, Dyer RB, Sadharabiriss A, Makgoba MW. Cardiac beriberi: a report of four cases. S Afr Med J 1987;72:283-285.

11. Patterson DL, Pang JA, Yardumian A, Davies R. Shoshin beriberi: an underdiagnosed condition? Intensive Care Med 1986;12:380-382.

12. Lopez GOD, Malvino ER, McLoughlin D, Osatnik J, Chavez ZMA, Pino C. Acute cardiovascular beriberi (Shoshin beriberi). Medicina (B Aires) 2002;62:331-334.

13. Meurin P. Shoshin beriberi, a rapidly curable hemodynamic disaster. Presse Med 2001;30:1256-1258.

14. Engbers JG, Molhoek GP, Arntzenius AC. Shoshin beriberi: a rare diagnostic problem. Br Heart J 1984;51:581-582.

15. Naidoo DP, Gathiram V, Sadhabiriss A, Hassen F. Clinical diagnostic of cardiac beriberi. S Afr Med J 1990;77:542-544.

16. Delorme N, Cornette A, Maurin P, Polu JM, Sadoul P. Shoshin beriberi with hiponatremia in a beer drinker. Presse Med 1986;15:2005-2009. 